\title{
Impact of Question Classification on Accuracy of Question Answering System
}

\author{
Divya Panicker \\ Research Scholar (Computer Engg.) \\ DYPIET (Pimpri) \\ Pune, India
}

\author{
Archana Chaugule \\ Professor (Computer Engg.) \\ DYPIET (Pimpri) \\ Pune, India
}

\begin{abstract}
Question answering system provides the user with functionality to get precise answer of the question articulated in their natural language. Question classification is a vital part of any question answering system. The accuracy of answer provide by question answering system heavily depends on the way question is classified. Accurate question classification leads to retrieval of exact answer in question answering system. Extracting whether a question is subjective or objective helps in analyzing the actual structure of answer expected by the users.
\end{abstract}

\section{Keywords}

Question answering, Question classification, Information retrieval, Natural Language Processing, neural networks.

\section{INTRODUCTION}

Question answering system (QAS) provides precise answer to question provided by the users. Most of the questions in QAS are in the form of natural language query, where user has freedom to provide their questions in their own natural language. Any question answering system basically consists of three parts question processing, answer retrieval and answer generation. In question processing users natural language question are parsed to formulate question in machine readable form using different approaches.

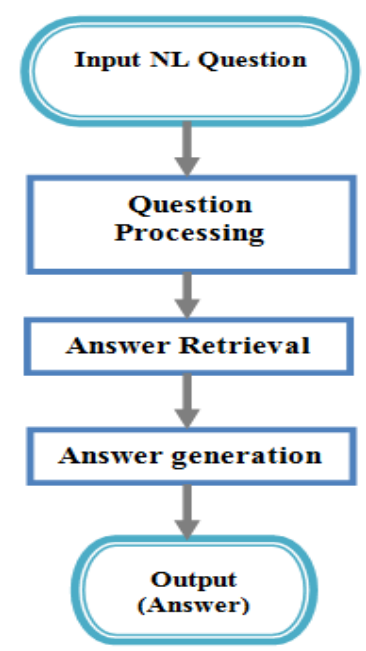

Fig 1.1 Basic architecture of question answering system

Then in answer retrieval candidate answers are extracted based in intermediate representation of question. Finally in answer generation phase user understandable precise and accurate answer is generated and provided to user.

An important part of most the question answering system is question classification. An improper question classification will lead to inaccurate answer. For example, the question of who discovered first Smartphone should be classified into the type of human. This classified information helps to understand that answer to the question should be name of some human being. In case question was classified incorrectly as location then answer retrieved will be irrelevant to the given question. Also information extracted through classification of question will help to slim down the search criteria and size to identify the correct answer from the available dataset.

Most of the question answering system uses manually written set of rules to map a question to its corresponding type. Up gradation and improvement of such rules is difficult task when number of rule increases.

Machine learning is a subset or form of artificial intelligence that provides computers around us with the capability to learn and explore without being need to program the system explicitly. Any Machine learning algorithm is set of programs which have the capability to teach and feed themselves to grow and explore new dimensions when it is exposed to new form and size of data.

Machine learning is form of data analysis which automates the process of analytical model building. Using algorithms which can iteratively learn from data, allows computers to find hidden insights from the data without being need to explicitly programmed to find logic to derive those insights.

Neural networks are a colony of nodes which are typically organized in layers. Every node in the network has set of activation type of functions in it. Input to any node in the network activates the node to process the node and pass it to other node.

Layers in the neural network can be broadly classified as input, hidden and output layers. Patterns are augmented to the network via the channel of input layer, which basically communicates to one or more than one hidden layers present in the networks. Hidden layers are the actual location in the network where the actual processing is of pattern is done via a system of weighted and unmeasured connections. The hidden layers are finally connected to an output layer where the answer is provides as shown in fig 1.2.

Most Artificial neural networks (ANN) contain some form of learning rule assigned to it which then alters the weights of the associated connections according to the different input patterns which is provided with the pattern. In a basic sense ANN learns by example same way human kids during their growth phase keep on learning things through various examples which exist around them. 


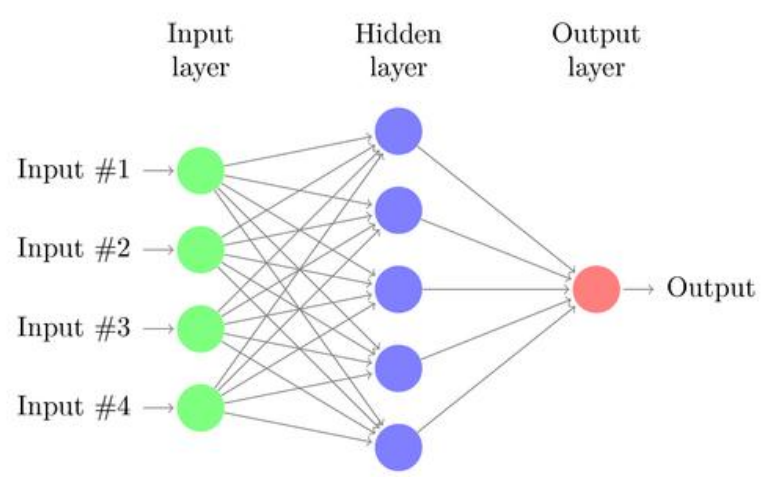

Fig 1.2 Neural network basic structure

Machine learning and neural network modules helps to overcome the limitation of rule based system where classification of question can be done on basis of different feature and would get more accurate results. Better advantage of machine learning or neural network approach is that one can focus on extracting insightful features from the data and rely on learning process to efficiently improve the accuracy and scalability of process. Also learned classifier is much more flexible and scalable to reconstruct than a manually constructed rule based system because it can be trained on a new taxonomy and rules at a very short span of time.

The paper presents, literature review of various question classification methodologies used in question answering system. In section 2, related work done for classifying question is presented in detail. Section 3 concludes the paper.

\section{RELATED WORK}

Various approaches have been used for classification of question as per respective question type. Classification of question as subjective or objective has benefited for retrieving relevant type of answer for question.

M. R. Morris et al. [1] has discussed the various fact of asking question by messages of social networking status. They have represented detailed study of data, the way question are asked, different types of question that can be asked and various user are motivated by asking question instead of using traditional approaches such as using tool like google. They have reported rate and class of answer received are superficial and reasons due to which people are willing to reply message to their near ones status.

S. A. Paul et al. [2] has discussed a way human are tagged with help of Amazon Mechanical Turk. It can be done by searching relevant search outcomes or values should worth of Wikipedia articles. For distinguishing and tagging different tweets as the way to ask question they have used Amazon Mechanical Turk. Author has even suggested the importance by mean of micro task for organizing bigger amount of studies and even it will include person brainpower task. Instructions that are given for completion of task were not able to identify by system. To explain the task to system is most tricky and hard as there might a lot disturbance while identifying data as question. Some of Question is asked without any answer expectation where as some question contain very less data and some of question contain insufficient data, so that question meaning is not understood.

D. Moy et al. [3] has explained the use of various machine learning technique and this technique can be used to differtiate question as informational and conversational, it will be done automatically. It even help to find a group of techniques that will has very good effect and the procedure to trained regarding categorical, linguistic, and social differences various type of question. They have introduces a classifier and this classifier will classify the quantitative features that are taken from the data that will be hardly different from their assumed classifier that will appear as conversational and informational questions. Author has also added some extra features to enhance the social network model, due to it performance of it will be improved. They have experimented with various grouping of human valuation and machine learning method to walk around with Question and answering questions. Author has revealed that conversational question asking is used to point out information quality.

A. Pal et al. [4] describe a novel concept of question temporality in QA communities where it is consider as a measure of how long the answers provided on a question are expected to be valuable. The duration when question is answered to question will help search engines and permit QA provider to look at appealing framework that will help to choice better one that are provided from user experience. It even guide to develop deeper understanding to increase question pro long existence of question. Their outcome proved that with the use of question vocabulary another featured results question temporality can be detected easily. Temporal question can be guessed using the feature such as non absolute time or text such as who, where, what

M. Jiang et al. [5] has presented comparatively new and appealing categorization issues of text that will group blog either as political, liberal or conservative. All this classification is based on political leaning. Author has categorized whether sentence is subjective, as sentence will have minimum more than one subjective clue and this clue is based on general inquirer dictionary and from recognized subjective sentence. From sentence opinion expression and other features were used to build classifier of political leaning. Outcome of their experiment on the blog account of political proved that by means of this feature from subjective has efficiently improved performance of classification. Even system will be capable to disclose the various opinion that will specify some of political learning.

P. Biyani et al. [6] has described an approach which uses supervised machine-learning. It will differentiate online forum as either subjective ones or objective one. Features generated from this approach as n-gram and POS tagging of data gives good outcome of treads in forum.

B. Li et al. [7] has discussed about the various issues that will automatically identify orientation of subjective question in QA communities. The have also search solution on supervised learning that will differentiate different feature which are needed for designing task. The experiment proved that characters which are not case sensitive 3 gram features will be much helpful to represent their task. Even they even exploited the answer which is given from different user for a given question so that accuracy for predictions is improved. Method used by the author gives better result than Naîve base.

L. Chen et al. [8] have introduced a model which will categorized the question into three categories on the basis of their considered user as objective, social and subjective. To identify the way the question was asked, author has built a predictive model from machine learning. This model will take text and metadata feature. The outcome of system has grouped features into two groups where this features are not dependent conditionally. These features can be used to predict. In semi supervised learning structure they have taken two view so that 
it make large amount of question which are not labeled where as small set will be labeled manually so that question classification will be enhanced. In the introductory experiment outcome showed that simply pooling is not as much better as co training works.

J. Wiebe et al. [9] have proved that outcome generated from subjectivity classifier with help of annotated text which is used for training. Using supervised learning approaches the performance will be improved. Previously the art state in objective sentence classification is learned by extraction pattern which is connected with the objectivity and creating classifier of objective that will get significantly higher recall.

C. Lin et al. [10] have described model for subjectivity detection LDA so that sentence level subjectivity classification is done. Existing approaches are different as they require label copra or linguistic pattern extraction. Author has viewed the issues as weakly supervised generative model learning where subjective or neural words are not dependent as input to the system. This model will evaluate the dataset of MPQA.The previous outcome proved that excluding Objective call, SubjLDA performed well LDA over all other evaluations metrics. Comparing to other approaches this introduced approach is much better one and it is proved with bigger training data set. It was observed that by adding more subjective words will produce much better results. The performances can be increase by adding extra neural words.

Y. Liu et al [11] has bring up an issue in collaborative question answering community of predicting information seeker satisfaction, where user attempted to predict whether a question will fulfilled the answer submitted by the member of communities. In this author has introduced a prediction model and even build up a variety of context, structures and features that are focused on community for completion of their task. Their experiment outcome that are received from a large scale evaluation using numbers of question and user reviews. It has also showed the possibility for modeling and predict whether user is satisfied. Author has proved a better result by doing a lot of investigation on the communication and pattern on seeking information about question answering community and correlating it with information seeker..

Z. Liu. et al [12] has introduced a model with an objective that user communication and mutual support in social question answering will get enhanced. The relationship between eleven extrinsic feature and rate of response for question was analyzed by author. Author has used statical methods multiple regression and weibo as data source. They has studied eight feature that has better effect on response rate. The feature that are considered by user are no of follower for questioniers, posting frequency, in question no of at-mention used, for an account whether a question will include any atmention, various hashtags, various emotions, timing and topic for posing of question. The acceptance of atmention verify account as well as hastag, both of them can have negative influence on the replied answer and it is exact opposite to hypothesis. Posting frequency has disapproval and it can be based on criteria variable. They have even discussed that problem can be discussed freom two point of view and it can have impact on the usability of social question answering. From SNS provider they haven't suggested an offical usage and even hastag can be benefit in the context of social Q\&A,visibility of question can not be increased.

Anderson et al [13] has proved a better way so that user can focus from one off answer to a group that has a process for knowledge creation.In this they have considered all question together so that entire collection of answer act as init for fundamental analysis. Whereas previously all work are done in question answer characterization. They have consider the different activity of community that will shape the set of answer. While considering different activities both answer and voter that are arrived during that time.Even it consider where those dynamic activity has any evevtual outcome.they have showed that understanding for such properties will be applicable for predicting several quantity which may include value for long term question as well as anwser for that particular question.

\section{CONCLUSION}

A question answering system which consists of an accurate question classification system provides much accurate answers for the given question. Classifying the question as subjective or objective helps to identify the nature of question type and target the input question to retrieve related subjective or objective form of answers from the available dataset. Classifying the question with the help of machine learning and neural network library's improves the accuracy of question classification where multiple features can be taken in consideration.

\section{REFERENCES}

[1] M. R. Morris, J. Teevan, and K. Panovich, "What do people ask their social networks, and why?: A survey study of status message q\&a behavior," in Proc. SIGCHI Conf. Human Factors Comput. Syst., 2010, pp. 17391748.

[2] S. A. Paul, L. Hong, and E. H. Chi, "What is a question? Crowdsourcing tweet categorization," in Proc. SIGCHI Conf. Human Factors Comput. Syst., 2011, pp. 578-581.

[3] F. M. Harper, D. Moy, and J. A. Konstan, "Facts or friends?: Distinguishing informational and conversational questions in social Q\&A sites," in Proc. SIGCHI Conf. Human Factors Comput. Syst., 2009, pp. 759-768.

[4] A. Pal, J. Margatan, and J. Konstan, "Question temporality: Identification and uses," in Proc. ACM Conf. Comput. Supported Cooperat. Work,2012, pp. 257-260.

[5] M. Jiang and S. Argamon, "Exploiting subjectivity analysis in blogs to improve political leaning categorization," in Proc. 31st Annu. Int. ACM SIGIR Conf. Res. Develop. Inf. Retr., 2008, pp. 725-726.

[6] P. Biyani, C. Caragea, and P. Mitra, "Predicting subjectivity orientation of online forum threads," in Proc. Comput. Linguistics Intell. Text Process., 2013, pp. 109_ 120.

[7] B. Li, Y. Liu, A. Ram, E. V. Garcia, and E. Agichtein, "Exploring question subjectivity prediction in community QA," in Proc. 31st Annu.Int. ACM SIGIR Conf. Res. Develop. Inf. Retr., 2008, pp. 735-736.

[8] L. Chen, D. Zhang, and L. Mark, "Understanding user intent in community question answering," in Proc. 21st Int. Conf. Companion World Wide Web, 2012, pp. 823828 .

[9] J. Wiebe and E. Riloff, "Creating subjective and objective sentence classifiers from unannotated texts," in Proc. 6th Int. Conf. Comput.Linguistics Intell. Text Process., 2005, pp. 486-497. 
[10] C. Lin, Y. He, and R. Everson, "Sentence subjectivity detection with weakly-supervised learning," in Proc. 5th Int. Joint Conf. Natural Lang.Process., Nov. 2011, pp. $1153-1161$

[11] Y. Liu, J. Bian, and E. Agichtein, "Predicting information seeker satisfaction in community question answering," in Proc. 31st Annu. Int.ACM SIGIR Conf. Res. Develop. Inf. Retr., 2008, pp. 483-
[12] Z. Liu and B. J. Jansen, "Factors influencing the response rate in social question and answering behavior," in Proc. Conf. Comput. Supported Cooperat. Work, 2013, pp. 1263-1274.

[13] Anderson, Ashton, et al. "Discovering value from community activity on focused CQA sites: a case study of stack overflow."Proceedings of the 18th ACM SIGKDD international conference on Knowledge discovery and data mining.ACM, 2012 